\title{
Different types of skew deviation
}

\author{
Th Brandt, $M$ Dieterich
}

\begin{abstract}
Although all manifest skew deviations appear the same for the clinician, skew deviation can result from different combinations of dysconjugate vertical ocular deviations. Evidence is presented for three different types of skew deviation when it occurs as a feature of an ocular tilt reaction. In type 1 (utricle) there is upward deviation of both eyes with different amplitudes, as described for otolith Tullio phenomenon in humans. In Type 2 (dorsolateral medulla oblongata) hypertropia of one eye occurs while the other eye remains in the primary position, the hypothetical mechanism of skew deviation in Wallenberg's syndrome. In Type 3 (midbrain tegmentum) there is simultaneous hypertropia of one eye and hypotropia of the other eye, as described for electrical stimulation of midbrain tegmentum in monkeys and observed in clinical cases with a paroxysmal ocular tilt reaction.
\end{abstract}

Skew deviation, or the Hertwig-Magendie sign, is a relatively common supranuclear vertical divergence of the eyes that is associated with lesions in the posterior fossa, particularly those involving the brainstem tegmentum from the diencephalon to the medulla oblongata. ${ }^{1}$ The combination of skew deviation with cyclorotation has not yet been convincingly investigated, since Trobe $^{2}$ in his study on cyclorotation in acquired vertical strabismus excluded all cases of skew deviation with a positive Bielschowsky's head tilt test. In a preliminary study we found supranuclear (monocular or binocular) cyclorotation in 48 out of 67 patients with acute brainstem lesions and many of them were associated with vertical divergence of the eyes. It is well recognised that the diagnostic localising value of skew deviation is limited and the question is justified whether skew deviation is a clinical entity. It may be due to: hypertropia of one eye while the other eye maintains the primary position; hypotropia of one eye while the other eye maintains the primary position; simultaneous hypertropia of one eye and hypotropia of the other eye; concurrent upward or downward deviation of both eyes but with different amplitudes.

For the clinician, however, all manifest skew deviations appear the same since one eye assumes the primary position of gaze in order to provide fixation.

Evidence is presented that skew deviation- when it occurs as a characteristic feature of ocular tilt reaction (OTR)-can result from different mechanisms. OTR, a synkinesis of lateral head tilt, skew deviation and ocular torsion $^{3}$ is based upon utricular as well as vertical semicircular canal input. It is mediated by graviceptive pathways from the labyrinths via ipsilateral ponto-medullary vestibular nuclei crossing to the contralateral rostral midbrain tegmentum. ${ }^{4}$ OTR can be caused by lesions (or electrical stimulation) of the utricle or the graviceptive pathways. Three types of skew deviation can be delineated on the basis of animal experiments and clinical observations in OTR. These types can be attributed to lesions at different sites from the utricle to the dorsolateral medulla and midbrain tegmentum.

\section{Type 1: Upward deviation of both eyes with} different amplitudes (utricle)

This pattern of vertical ocular divergence was described in a patient with otolith Tullio phenomenon due to a subluxated stapes footplate. ${ }^{5}$ The Tullio phenomenon consists of pathological sound-induced vestibular symptoms in patients with perilymph fistulas. Surgical exploration of the middle ear of our patient revealed the loose stapes footplate with the hypertrophic stapedius muscle causing pathological large amplitude movements during the stapedius reflex. Sound stimulation of

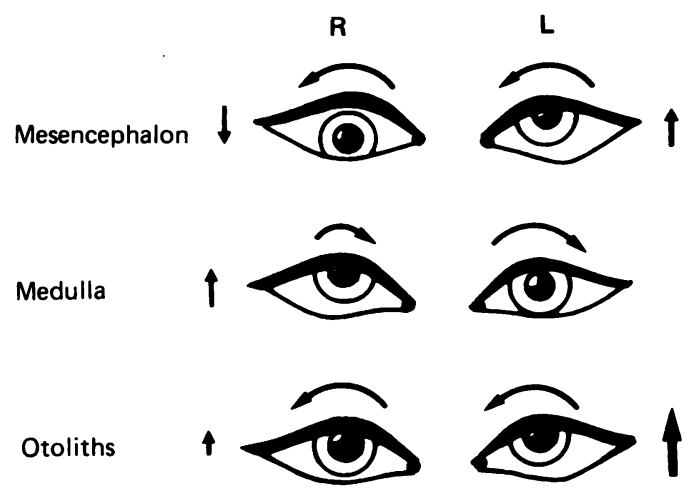

Figure Different types of skew deviation have been described as a feature of ocular tilt reaction (OTR) depending on the site from which $O T R$ is elicited. Simultaneous hyper-and hypotropia have been described for paroxysmal OTR in stimulation of the midbrain tegmentum in the monkey and humans; monocular hypertropia causes skew deviation in dorsolateral medullary lesions in humans; concurrent upward deviation of both eyes with different amplitudes was observed with mechanical stimulation of the otoliths in observed with mechanical stimulation of the otoliths indicated by the curved arrows; side of stimulation or lesion is left. 
the affected ear induced paroxysms of OTR because of a non-physiological mechanical stimulation of the otoliths. Both eyes showed an anticlockwise rotatory-upward deviation with skew deviation caused by dysconjugate large deviation of the ipsilateral eye.

Type 2: Hypertropia of one eye (dorsolateral medulla oblongata)

In dorsolateral medullary infarctions (Wallenberg's syndrome), OTR is characterised by dysconjugate ocular torsion with predominant excyclotropia of the ipsilateral eye. This is best explained by a circumscribed lesion of the ascending pathway of the ipsilateral posterior semicircular canal. ${ }^{4}$ The posterior canal has excitatory connections to the ipsilateral superior oblique and the contralateral inferior rectus muscles. A lesion of this pathway (which also conveys otolith input) would cause excyclotropia of the ipsilateral eye as well as skew deviation with elevation only of the contralateral eye. If the lesion damages both posterior and anterior canal pathways, then a complete OTR would result with hypotropia of the ipsilateral eye, hypertropia of the contralateral eye and conjugated ocular torsion towards head tilt. ${ }^{4}$

Type 3: Simultaneous hypertropia of one eye and hypotropia of the other eye (midbrain tegmentum)

Westheimer and Blair ${ }^{3}$ report an OTR with upward deviation of one eye and downward deviation of the other during electrical stimulation of the rostral midbrain tegmentum in the region of the interstitial nucleus of
Cajal of the monkey. This fits the clinical case reports on paroxysmal OTR in patients with different upper brainstem lesions (multiple sclerosis, ${ }^{6}$ brainstem abscess ${ }^{7}$ ).

Thus at least three different types of skew deviation, which are associated with cyclorotation and ocular tilt reaction, can be differentiated. Topographic attribution to peripheral or central vestibular structures is preliminary and may be too dogmatic. Electrical stimulation of the utricle nerve in the $\mathrm{cat}^{8}$ produced type 3 rather than type 1 skew deviation. It is most probable that there are more types which are not yet identified. About $12 \%$ of the patients with skew deviation exhibit a confusing alternating hypertropia on lateral gaze to either side or a simultaneous slowly alternating skew deviation. ${ }^{9}$

1 Keane JR. Ocular skew deviation: analysis of 100 cases. Arch Neurol 1975;32:185-90.

2 Trobe JD. Cyclodeviation in acquired vertical strabismus. Arch Opthalmol 1984;102:717-20.

3 Westheimer G, Blair SM. The ocular tilt reaction-a brainstem oculomotor routine. Invest Ophthalmol 1975;14: 833-9.

4 Brandt Th, Dieterich M. Pathological eye-head coordination in roll: tonic ocular tilt reaction in mesencephalic and tion in roll: tonic ocular tilt reaction in mesenc

5 Dieterich M, Brandt Th, Fries W. Otolith function in man. Results from a case of otolith Tullio phenomenon. Brain Results from a case

6 Rabinovitch HE, Sharpe JA, Sylvester TO. The ocular tilt reaction. A paraoxysmal dyskinesia associated with elliptical nystagmus. Arch Opthalmol 1977;95:1395-8.

7 Hedges TR, Hoyt WF. Ocular tilt reaction due to an upper brainstem lesion: paroxysmal skew deviation, torsion, and oscillation of the eyes with head tilt. Ann Neurol 1982; 11:537-40.

8 Suzuki JI, Tokumaso K, Goto K. Eye movements from single utricular nerve stimulation in the cat. Acta Otolaryngol 1969;68:350-62.

9 Keane JR. Alternating skew deviation: 47 patients. Neurology 1985;35:725-8. 